\title{
An Opportunistic MAC in Multichannel Multiradio Wireless Ad Hoc Networks
}

\author{
Feng Chen ${ }^{\dagger}$, Hongqiang Zhai ${ }^{\ddagger}$ and Yuguang Fang ${ }^{\dagger}$ \\ ${ }^{\dagger}$ Department of Electrical \& Computer Engineering, University of Florida, Gainesville, Florida 32611 \\ ${ }_{\ddagger}^{\ddagger}$ Philips Research North America, Briarcliff Manor, New York 10510 \\ E-mail: chenf@ecel.ufl.edu, hong.zhai@philips.com,and fang@ece.ufl.edu
}

\begin{abstract}
Opportunistic scheduling has been known as one of the possible ways of exploiting multi-user diversity, which comes from the time-varying propagation characteristics and broadcast nature of wireless radios, to improve communication efficiency and reliability. Multi-channel multi-radio technology has offered great opportunity in designing wireless network MAC protocol to utilize all kinds of resource diversities. Other than multi-user diversity, we identify the unique multi-radio diversity which can be exploited in multi-radio wireless network to improve network throughput performance. The proposed Opportunistic Multi-radio MAC (OMMAC) takes advantage of multi-radio diversity to increase the spectrum usage as much as possible. Extensive NS2 simulation results demonstrate that OMMAC significantly improves the network throughput in both single and multi-hop wireless networks.
\end{abstract}

\section{INTRODUCTION}

Last few years have witnessed the tremendous endeavor invested by the industry in pushing multi-channel multi-radio technology [16][17][18]. As the products with multiple radios with the ability of switching channel dynamically become available, the question of how to intelligently use multiple channels and multiple radios in designing more efficient and reliable wireless network has triggered a lot of research work in multi-channel multi-radio MAC designs [2][5][6][7][11][12]. The existence of multiple channels and multiple radios has greatly expanded manageable resource space, i.e., channel, radio, user, and time, for improving network throughput performance. Inspired by multi-user diversity in the seminal paper [9], we specifically identify multi-radio diversity in multi-channel multi-radio wireless networks and propose OMMAC to improve throughput performance by exploiting multi-radio diversity.

Opportunistic transmission schemes in general can be perceived as a way to utilize the physical-layer feedback from multiple sources to improve performance through medium access control [14], packet scheduling [13], and rate adaptation [1]. The proposed OMMAC can be regarded as a natural integration of these three approaches to maximize the extent of exploiting resource diversities. Due to different interference level, channel fading statistics, and geographical difference of users, variable maximum data rates can be supported on different transmission links over the same channel. OMMAC schedules the transmission on per-channel basis, i.e. selecting the best transmission pair for each channel, rather than perpacket basis which selects the best channel for transmission, every time a packet gains access to the medium [3].

In order to exploit multi-radio diversity, OMMAC uses multicast RTS, virtual multi-CTS and channel-based packet scheduling to collect receiver-measured channel condition over several candidate transmission links. The above mentioned techniques make it possible to measure several channels simultaneously and send back a set of quality information at one time. Moreover, as a middle layer between IEEE802.11 MAC and the logical link control layer, OMMAC keeps modifications in IEEE802.11 as few as possible.

OMMAC is closely related to two categories of research work. One is multi-channel multi-radio MAC design, the other one is MAC protocol exploiting resource diversities. Due to the technical limitation of the number of radios on each host, many previous work of MAC design focus on how to efficiently use multiple channels with one or two transceivers [6][7][11][12]. None of them can be easily tailed to achieve the goal of scheduling multiple transmissions among several available radios, if there are, on a single node. For practical multi-radio MAC schemes in multi-hop ad hoc wireless network with the aim of improving network capacity, the most related work to OMMAC would be [4], which proposes a multi-radio unification MAC protocol to coordinate the operation of multiple wireless network cards. However, the channel assignment is fixed there which limits the extent of using local spectrum. Adopting the similar approach of adding a unified layer above original MAC layer, OMMAC tries to push forward the idea of improving spectrum usage efficiency by considering the dynamic channel selection for several available radios simultaneously. Compared with opportunistic transmission schemes [1][3][9], OMMAC provides a way of exploiting multi-radio diversity, which achieves greater throughput improvement as shown in NS2 simulations.

The remainder of this paper is organized as follows. First in Section II, we introduce the key ideas for OMMAC. Second in Section III, we describe the detailed protocol design issues. The performance evaluation through NS2 is given in Section IV. Finally, we draw the conclusion in Section V.

\section{DESIGN INSPRIATIONS}

\section{A. Multiple MAC Diversity Issues}

The multiple diversities come from geometrical difference among receivers, interference level, fading characteristics and 


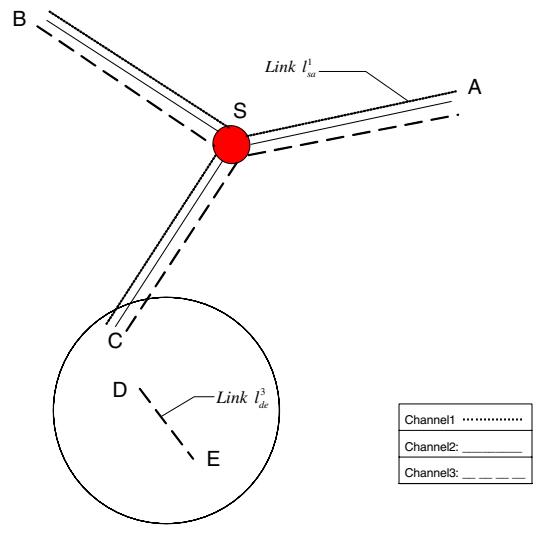

Fig. 1. Illustration for link diversity, channel diversity, and multi-radio diversity. Node $S$ is the source node, node $A$, node $B$, node $C$ are receivers. There is an ongoing transmission between node $D$ and node $E$ on channel 3. The figure shows differences in geographical location and interference level which give rise to multiple diversities.

transmission medium. We illustrate three kinds of diversities through the scenario shown in Fig. 1.

Link Diversity : Given a channel on a fixed frequency, the link diversity exists in the scenario in which there are several potential receivers for transmission. At one time, the quality of a link between a pair of a certain source and its next-hop neighbor is better than others. For example, the link set $\left\{l_{s a}^{i}\right.$, $\left.l_{s b}^{i}, l_{s c}^{i}\right\}$ can represent a source of link diversity, where $l_{s a}^{i}$ is defined as the link between $S$ and next-hop $a$ on channel $i$. We denote it as a link diversity set.

Channel Diversity: Given several available channels on orthogonal frequencies or codes, several transmissions can be carried on simultaneously in different channels without interfering with eac other. For example, the link set $\left\{l_{s a}^{1}, l_{s a}^{2}, l_{s a}^{3}\right\}$ can represent a source of channel diversity. We denote it as a channel diversity set.

Multi-radio Diversity: Given several available channels and more than one radio on a single node, the multi-radio diversity arises in the scenario where different geographically located users experience different link qualities. For example, the link set $\left\{l_{s j}^{i}: j \in(a, b, c), i \in(1,2,3)\right\}$ can represent a source of multi-radio diversity. We denote it as a multi-radio diversity link set. From the definition, it is clear to see that the multiradio diversity set is the outer product of the link diversity set and the channel diversity set, thus providing a larger space for exploiting the diversities in the network.

\section{B. Channel-based Packet Scheduling}

In this section, we illustrate how OMMAC could improve local spectrum usage by considering the total throughput of locally available radios on a single node. One way to utilize the multi-radio diversity is to select the best subset of the multi-radio diversity set for the transmission to maximize local throughput. We denote the packet with next hop address $A$ as $P_{a}$. Here the next-hop addresses also indicate their positions in the queue in an alphabetical order. Take Fig. 1 for instance. Suppose there are packets $\left\{P_{a}, P_{b}, P_{c}\right\}$ in the outgoing queue

\begin{tabular}{|c|c|c|c|}
\hline Next hop Data rate & Channel 1 & Channel 2 & Channel 3 \\
\hline A & 36 & 24 & 24 \\
\hline B & 54 & 36 & 24 \\
\hline C & 36 & 54 & 6 \\
\hline
\end{tabular}

Fig. 2. Illustration for localized optimization. For each next-hop address (corrsponding to a packet), the link quality on different channel may be different. For each channel, the link quality with different receivers may be different. From Fig. 1, due to the ongoing transmission, the maximum supportable datarate on $l_{s c}^{3}$ is only 6 . The horizontal ellipse represents packetbased scheduling, while vertical ellipse represents channel-based scheduling.

of node $S$. The current link qualities are described in Fig. 2. If $S$ selects the best channel whenever a packet gets the access for transmission, $S$ has to select link $l_{s a}^{1}, l_{s b}^{2}$ and $l_{s c}^{3}$ sequentially for packets $P_{a}, P_{b}$ and $P_{c}$. The total throughput for node $S$ at that time is $78 \mathrm{Mb} / \mathrm{s}$. We refer to it as packetbased packet scheduling. In OMMAC, with the objective of improving local spectrum usage efficiency, the optimal packet scheduling policy would be the link subset $\left\{l_{s b}^{1}, l_{s c}^{2}, l_{s a}^{3}\right\}$ or $\left\{l_{s b}^{1}, l_{s c}^{2}, l_{s b}^{3}\right\}$. In this case, the total throughput is $132 \mathrm{Mb} / \mathrm{s}$. We call it as channel-based packet scheduling. In Fig. 2, it is clear to see that channel-based packet scheduling is optimized for each channel, while packet-based packet scheduling is done for each packet. When applying the previous multi-channel schemes [2][5][6][7][11][12] directly into multi-radio systems, they usually fall into the category of packet-based scheduling.

Would local throughput optimization help improve aggregate throughput in multi-hop wireless network? It is believed that local optimization would not necessarily lead to the global optimization. However, solving global optimization of the throughput of network requires perfect knowledge of traffic pattern, network topology and interference distribution. Even with all the knowledge of the above, the problem itself is NP hard [4]. Thus, to the best knowledge of the authors, almost all the MAC protocols have not achieved the global optimal solution of maximizing total throughput of the network. In some sense, all of the related research works such as [1][3][4] try to improve the local throughput instead. Many show the performance improvement in network throughput through experiments or simulations. We will show the performance evaluation in Section IV. Numerical performance analysis is omitted due to space limit but provided in [19].

\section{Opportunistic MAC using Multiple Radios}

From the above discussion, it should be clear now that in OMMAC the "opportunities" lie in multi-radio diversity. The question of how to obtain the information about link qualities in multi-radio diversity link set is the focus of design issues of OMMAC. In OMMAC, the mechanism of sending RTS to probe the channel condition is used for collecting link quality information. However, instead of using unicast RTS, OMMAC uses multi-cast RTS on available channels to candidate receivers over several available radios. The multi-cast 


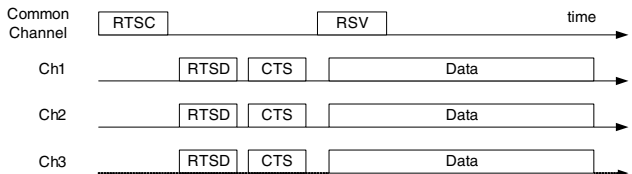

Fig. 3. Time line for OMMAC. Assume the current transmission uses 4 radios at one time. During the process of RTSC-RTSD-CTS, the sender is able to collect channel information on 9 links if the negotiation process is successful.

RTS contains information for coordinating receivers to simultaneously monitor several channels and send back CTS with as much information as possible. Using a single CTS to carry back the link quality information for several channels greatly enhances sender's ability to achieve the optimal scheduling according to the current link states. Moreover, it is worth to point out that one RTS and CTS pair in IEEE 802.11 MAC can only provide quality information on one link. Therefore, IEEE 802.11 MAC needs much more pairs of RTS and CTS to collect the same information that OMMAC does.

\section{Protocol Description}

In this section, we propose a novel Opportunistic Multi-radio MAC (OMMAC) protocol to utilize the multi-radio diversity. Before elaborating the protocol in details, we first summarize our assumptions here. $N$ channels are available for use in a multi-hop multi-rate wireless network and all channels have the same bandwidth. None of the channels overlaps, so the packets transmitted on different channels do not interfere with each other. One of the channels is chosen to be the common channel with default frequency. Each communication node is equipped with 1 to $M$ half-duplex transceivers. Every transceiver is capable of switching its channel dynamically and the channel switching time $224 u s$ is [11].

\section{A. RTS on Common Channel(RTSC) and Multi-cast RTS on Data Channel(RTSD)}

In OMMAC, one channel with default frequency is chosen to be common channel. Common channel is known being able to alleviate hidden terminal problems [7]. Schemes without common channel [10][11] usually have the synchronization issue which needs extra signaling overhead. Since there is usually one negotiation for more than one transmission in OMMAC, the common channel saturation [10] problem should be alleviated.

To start a transmission, a sender transmits a RTSC on the common channel. RTSC is particularly important since it contains the channel list and the next-hop list which are important information to regulate receivers' behavior and establish communication channels between them.

- Channel list construction: First the sender checks its available channels and radios whose numbers are denoted as $N_{s}$ and $M_{s}$. We denote the set of total channels and radios at the sender side as $C_{s}$ and $R_{s}$. We denote the available channel set of source $\mathrm{S}$ as $c_{s}$, where $c_{s} \subseteq C_{s}$ and $\left\|c_{s}\right\|=N_{s}$. Similarly we denote the available radio set as $r_{s}$ where $r_{s} \subseteq R_{s}$ and $\left\|r_{s}\right\|=M_{s}$. The number of radios that the node will use for data transmission is at most $\min \left(N_{s}, M_{s}\right)$, denoted as $\min _{s}$. Here the sender simply randomly choose $\min _{s}$ channels from its available channel pool $c_{s}$ to form the channel list in RTSC.

- Next-hop list construction: The next-hop list contains the addresses of candidate receivers. Denote the packet set in the queue of node $S$ as $P_{s}$ where $P_{s}=\left\{p_{s}^{i}: i \in\right.$ $(1,2, \ldots, q)\}$ and $q$ is the queue length. The next-hop address set in the queue is defined as the next-hop index set $D_{s}=\{1,2,3, \ldots, d\}$ where $d$ is the maximum number of different next hop addresses in the queue. In order to fully exploit the multi-radio diversity as much as possible, the basic rule for selecting receiver candidates is

$$
\begin{aligned}
& \max \left|I_{s}\right| \\
& \text { s.t. } \\
& P_{s d}=\left\{p_{s i}^{j} \mid i \in I_{s}, p_{s i}^{j} \in P_{s}\right\}, \\
& \left|P_{s d}\right|=\min _{s}, \\
& I_{s} \subseteq D_{s} .
\end{aligned}
$$

Here, the sender tries to choose as many packets with different next-hop addresses as possible under the constraint of the maximum number of packets to schedule is $\alpha \mathrm{min}_{s}$. The greater the $\alpha$ is, the larger the multi-radio link set is and possibly longer waiting time for the feedback from receivers is. In OMMAC, we let $\alpha=1$.

As a side note, there is one to one mapping between the elements in the next-hop list and the channel list for coordinating the transmission of CTS, i.e., the $i_{t h}$ receiver in the next-hop list will send CTS on the $i_{t h}$ channel in the channel list. We leave it in Section III-B for more detailed description about channel selection for CTS.

RTSD is actually used as a probing message on the channel where RTSD is transmitted. Receivers who hear RTSD on the corresponding channels will be able to measure the channel quality and provide channel information at receiver side. Notice that each RTSD contains the same next-hop list. The multicast property of RTSD enables the measurement of channel quality at different receivers who may experience different link qualities on different channels or at different locations. In the case that a legitimate receiver may not hear RTSC but happen to hear RTSD on one or several data channels (assuming the radio will monitor the channel if it is idle), the receiver will send back CTS according to RTSD. The channel list in RTSD will serve the same way as RTSC to enable the channel selection for sending back CTS.

\section{B. Virtual Multi-CTS}

Virtual Multi-CTS is a single CTS but containing the amount of quality information for several channels which the node sending CTS was able to measure. Denote the channel set that the $i_{t h}$ receiver in the next-hop list of RTSC is able to measure as $C_{r_{i}}$. Apparently, $C_{r_{i}} \subseteq C_{s}$. If the number of available radios 
at the $i_{t h}$ receiver, denoted as $M_{r_{i}}$, is equal to the length of the channel list in RTSC, then $C_{r_{i}}=C_{s}$. Otherwise, receiver has to choose a subset of channels in $C_{s}$ to listen to. The first priority is given to the $i_{t h}$ channel in the channel list in RTSC because the $i_{t h}$ receiver should reply a CTS on that channel, denoted as $C_{c t s}$. If the same address has more than one position in RTSC's next-hop list, then CTS is sent on the channel with the smallest index which will also be the first choice of the receiver to tune radio to. The second priority is given to the channels that available radios are already sitting on. This rule tries to minimize the channel switching cost as much as possible. For the rest of available radios, the receiver randomly tunes the radios to channels which are in the channel list in RTSC. Since one receiver can listen to several channels simultaneously with multiple available radios, each receiver replies with a CTS frame containing the values of supportable data rates for channels in set $C_{r_{i}}$.

What happens if RTSD is not heard on the channel $C_{c t s}$ ? Even though we make sure that receiver will first tune one of its available radios to $C_{c t s}$, it is still possible that the link quality on $C_{c t s}$ is so poor that RTSD can not be heard. Then if it is the case, the receiver is going to skip channel $C_{c t s}$ and switch the radio to the next channel in the channel list in RTSC. If the channel is idle ${ }^{1}$, then the receiver will send CTS through that channel. The maximum number of channel skipping is a parameter which is a tradeoff between CTS waiting time and the amount of information the sender wish to get. According to channel skipping rule here, receivers are coordinated to transmit on distinct channels if they need to participate in sending CTS once again.

\section{Opportunistic Packet Scheduling for Multiple Radios}

Based on the information carried by CTS, a sender starts packet scheduling with the goal of optimizing local throughput of all its available radios. To put it straightforward, the problem is formulated as below:

$$
\begin{aligned}
& \max \sum_{i} \sum_{j} x_{j}^{i} q_{j}^{i} \\
& \text { s.t. } \\
& \sum_{i} \sum_{j} x_{j}^{i} \leq \min _{s}, \forall i \in c_{s}, j \in D_{s} \\
& \sum_{i} x_{j}^{i} \leq\left|C_{r j}\right|, \forall i \in c_{s} \\
& \sum_{j} x_{j}^{i} \leq 1, \forall j \in D_{s} \\
& x_{j}^{i} \in\{0,1\}, \forall i \in c_{s}, j \in D_{s}
\end{aligned}
$$

Where $q_{j}^{i}$ is the supportable maximal data rate on link $l_{s j}^{i} . x_{j}^{i}$ is packet scheduling policy at for the $j_{t h}$ next hop receiver on the $i_{t h}$ channel. The value of $x_{j}^{i}$ are either 0 or 1 , with 1 for scheduling transmission on the $i_{t h}$ channel for packets to the $j_{t h}$

\footnotetext{
${ }^{1}$ channel sensing time is assumed to be short enough compared with CTS transmission.
}

next hop. From the problem formulation, it is clear to see that packet scheduling is done on per-channel basis, that is, for each channel, choose a transmission pair with the best link quality for current transmission. It is different from the approach many multi-channel schemes adopt, which is to select a relativegood channel for each transmission pair. As explained earlier in Section II-B, the approach we use here takes better usage of all available spectrum usage. Another recent parallel work taking the same perspective of view from per-channel basis is [8], however it does not support multirate transmission and does not consider the local throughput optimization problem.

The above formulation is a combinatorial optimization problem. Since the numbers of channels and radios are usually small, we use recursive brute-force search to solve the problem. The algorithm is executed by the sender to determine the packet scheduling strategy.

\section{Channel Reservation and Channel Usage List Update}

Carrying the information of the channel assignment and corresponding channel occupying time, RSV(channel ReSerVation packet) tells all the neighboring nodes that which channel will be occupied and how long it is going to be occupied.

All the nodes will update their local Channel Usage List according to RSV. Local channel usage list records channel occupation time and the receiver for the current transmission for each channel. Channel occupying time is used to set the network allocation vector (NAV) for each Channel. Through Channel usage list, a sender could infer how many radios available at each destination, which assists sender for packet scheduling. If there is no available radio at a destination, sender delays the packets destined to those nodes. Sender also chooses to transmit packets to nodes with more available radios than those with less available radios for a certain period of time.

From the above, it should be clear that OMMAC is able to exploit multi-radio diversity as much as possible through multicast RTS, virtual multi-CTS, RSV, and local channel-based packet scheduling while keeping the communication overhead low.

\section{E. Illustrative Example}

In this section, we outline the procedure of the OMMAC through an example as shown in Fig. 1: Suppose $S, A, B$, $C$ are the nodes with 3 available radios for data channels. In source $S$ 's outgoing queue, there are packets with next-hop addresses $A, B, C$. For $S$ to initiate a transmission, $S$ first multicasts a RTSC frame on the common channels. The RTSC frame includes the channel list indicating that $\operatorname{ch} 1, \operatorname{ch} 2, \operatorname{ch} 3$ are chosen from the available channels by $S$. It also includes the next-hop address list consisting of $A, B, C$ for the packets in its queue. Through the coordination of RTSC, nodes $A, B$ and $C$ switch their radios to listen to all these three channels ch $1, \operatorname{ch} 2$ and $\operatorname{ch} 3$. By listening to the following RTS on the three data channels(RTSD), $A$ is able to measure the instant channel qualities for link set $\left\{l_{s a}^{1}, l_{s a}^{2}, l_{s a}^{3}\right\}$ and sends back a single virtual multi-CTS with the channel qualities for several links on one of channels among $\{\operatorname{ch} 1, \operatorname{ch} 2, \operatorname{ch} 3\}$. So does nodes 
$B$ and $C$. In this way, the link information collected by node $S$ could be in Fig. 2. As explained in Section II-B, the local spectrum usage is minimized by carrying out the channel-based packet scheduling which results in subsequent data transmission on the links $\left\{l_{s b}^{1}, l_{s c}^{2}, l_{s a}^{3}\right\}$ or $\left\{l_{s b}^{1}, l_{s c}^{2}, l_{s b}^{3}\right\}$. Then RSV is sent on the common channel to inform other nodes in the neighborhood to update their local channel usage lists. Since the receivers $A$, $B, C$ have already tuned their radios on channels indicated by RTSC, the reception of data packets can take place at the same time of transmitting RSV. The timeline for OMMAC is shown in Fig. 3.

\section{Performance Evaluation}

In this section, we use NS2 simulator to evaluate the performance of OMMAC. We extend NS2 to support multiple rates as in [15] and the Ricean propagation model as in MOAR [3]. First, we begin with single-hop topologies to study the main protocol properties and illustrate the impact of different parameters-number of radios, number of flows-on throughput performance. Generally, as the number of radios or the number of flows increases, the multiradio diversity set becomes larger. The simulation shows that OMMAC has successfully coordinated multiple radios to takes advantage of those resource diversity to improve the network performance. Second, we study multi-hop topologies to verify the performance in wireless ad hoc network. We also compare the aggregate throughput per radio (including the common channel) performance of OMMAC with that of $\mathrm{MOAR}^{2}[3]$ and demonstrate that OMMAC significantly improves the throughput by exploiting the multi-radio diversity. In the simulation, there are total 11 nonoverlapping channels. Three different rates, 2, 5.5, and 11Mbps, are studied. As in MOAR, their transmission radii are 250, 200, and $100 \mathrm{~m}$, respectively. UDP traffic is used. Simulation time is 50 seconds in each run. Packet size is 1000 bytes.

\section{A. Single-hop rectangular topology}

In the single-hop topology, all nodes are within the transmission range of each others. To separate the influence of topology, we start with a rectangular topology as shown in Fig. 4 to study the impact of different parameters on the performance. We change the parameters including the number of radios, the number of flows, and the channel fading parameter in the simulation to observe their impact on throughput.

Impact of number of radios: Here we use different number of radios to investigate the relationship between the number of radios and the throughput. There are eight flows generated from the source node $S$ to the other eight nodes. Fig. 5 illustrates that the throughput increases along with the number of radios. This indicates that OMMAC has successfully coordinated the

\footnotetext{
${ }^{2}$ MOAR is a MAC protocol for multi-channel and multi-rate enabled wireless ad hoc networks. It optimally skips channels to use frequency diversity and achieved improved throughput performance compared with OAR[1]. We choose MOAR as a representative of opportunistic multi-channel MAC. Considering it is for single radio, We compare the performance on per-radio base. For multi-radio opportunistic MAC, so far we do not have any pre-existing online implementation that we can use as a comparison.
}

available radios for transmission to improve the local spectrum usage.

Impact of number of flows: In this simulation, we set the number of radios as four. From Fig. 6, we see a dramatic increase in throughput when the number of flows increases from 1 to 2 , which results from the unique feature of packet scheduling in OMMAC. When the outgoing queue has many packets with different next-hop addresses, the multi-radio diversity consists of a large set of link diversities, which gives OMMAC the opportunity to optimally schedule the transmissions and improve the throughput. Due to the limitation of the extent of channel quality variation and the number of radios, the throughput becomes steady when the number of flows is close to the number of radios. Also notice that the per-radio throughput of OMMAC is greater than that of MOAR even though the single-radio OMMAC has fewer channels to schedule the transmissions than MOAR, the multi-radio diversity still offers performance improvement in per-radio aggregate throughput in OMMAC.

Impact of Ricean parameter $K$ : Here we use four radios in each node. There are still eight flows generated from the source $S$ to the other eight nodes. We tune the Ricean parameter $K$ from 0 to 6 . The larger the value of $K$ is, the smaller variation there is in channel quality. Fig. 7 shows throughput of both MOAR and OMMAC. Here the per-radio aggregate throughput in OMMAC is still greater than MOAR. Similar with MOAR, OMMAC's throughput increases when $K$ increases.

\section{B. Single-hop random topology}

In this subsection, we consider a random topology where the mobile nodes are uniformly distributed in a circular area with a diameter of $250 \mathrm{~m}$. We choose Ricean fading parameter $K$ equal to 4 . The throughput of both MOAR and OMMAC is shown in Fig. 8. The increase in aggregate throughput along with the number of flows again shows the benefit of exploiting multi-radio diversity. OMMAC improves the throughput by 2.33 times up to 8.91 times. Moreover, the per-radio aggregate throughput in OMMAC outperforms MOAR by average 1.4 times.

\section{Multi-hop random topology}

In this subsection, we study a multi-hop topology where mobile nodes are uniformly distributed in a $1000 \mathrm{~m} \times 1000 \mathrm{~m}$ rectangular area. We choose Ricean fading parameter $K$ equal to 4. We use AODV as the routing protocol. As shown in Fig. 9, OMMAC significantly improves the throughput compared with MOAR, and the gain is from 3.27 times to 11.22 times when the number of flows increases from 2 to 16 . Also the per-radio aggregate throughput in OMMAC improves as the number of flows increase and outperforms MOAR when the number of flows is greater than 4 . The first flow has zero throughput since they are disconnected in the simulated random topology.

From all the results above, we can see that OMMAC performs well in exploiting the multi-radio diversity. The aggregate throughput, both multi-radio and per-radio aggregate throughput, is improved than the previous work MOAR. 


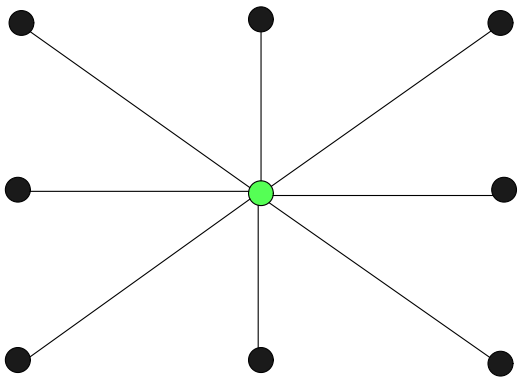

Fig. 4. Single-hop Topology: the size of the rectangle is $200 \mathrm{~m} \times 200 \mathrm{~m}$. $S$ is the source node.

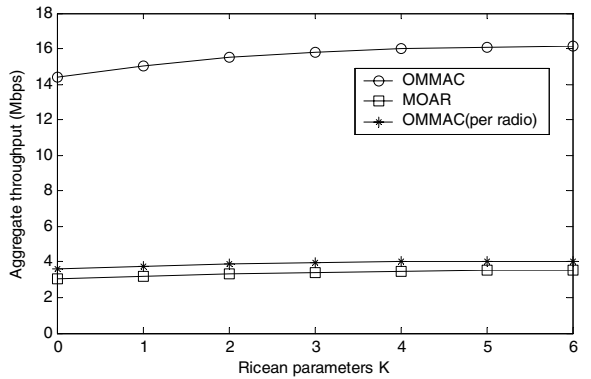

Fig. 7. Throughput of OMMAC as a function of the Ricean parameter $\mathrm{K}$ in the single-hop rectangular topology
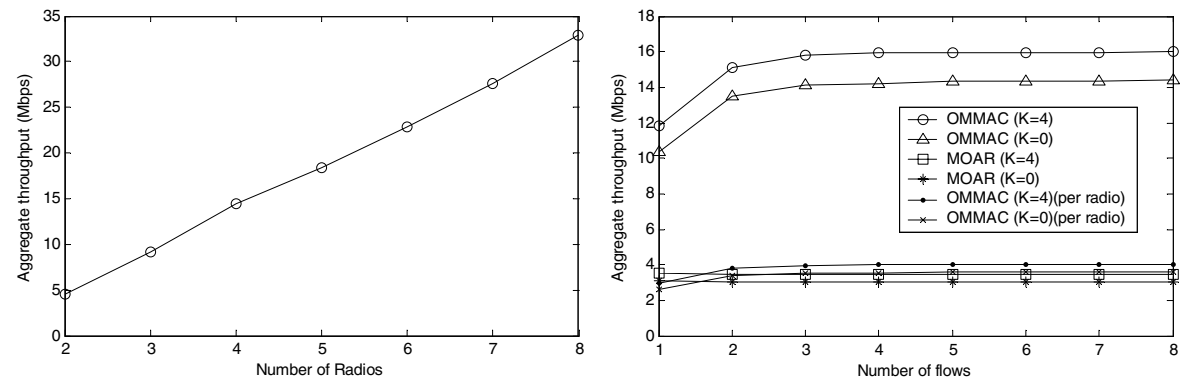

Fig. 5. Throughput of OMMAC as a function of number of radios in the single-hop rectangular topology

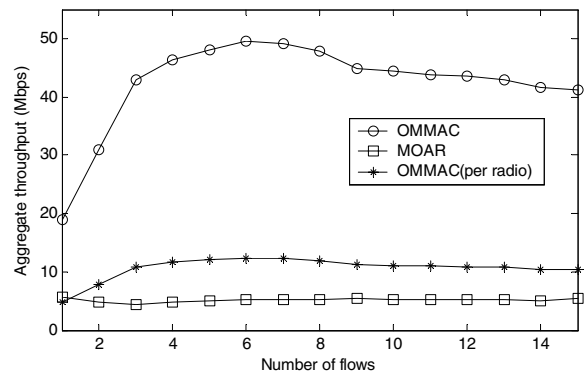

Fig. 8. Throughput of OMMAC as a function of number of flows in the single hop random topology in comparison with that of MOAR
Fig. 6. Throughput of OMMAC as a function of number of flows with different Ricean parameters compared with that of MOAR in the single-hop rectangular topology

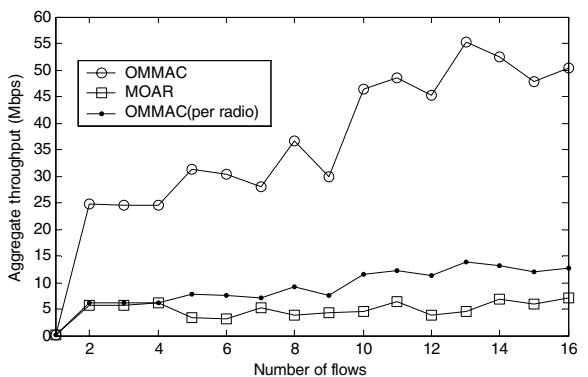

Fig. 9. Throughput of OMMAC as a function of number of flows in the multi-hop random topology in comparison with that of MOAR

\section{CONCLUSION}

In this paper, we specifically identify multi-radio diversity in multi-channel multi-radio wireless network and propose OMMAC as a way of exploiting multi-radio diversity through multi-cast RTS, virtual multi-CTS and channel-based packet scheduling. The existence of multi-radio diversity provides a larger space for selecting links with good channel quality for transmission. OMMAC plays great endeavor in coordinating multiple radios on each node to gather link quality information upon which it carries the channel-based packet scheduling by considering the total throughput of available radios. Extensive NS2 simulation results have shown that OMMAC has great improvement in the aggregate throughput per radio.

\section{REFERENCES}

[1] B. Sadeghi, V. Kanodia, A. Sabharwal, E. Knightly, "OAR: an opportunistic auto-rate media access protocol for ad hoc networks", inwireless networks, Volume 11, Issue 1-2 (January 2005), Pages: 39 - 53, 2005 ISSN: 1022-0038.

[2] J. Mo, H. Wilson So, J. Walrand, "Comparison of multi-channel MAC protocols", in International Workshop on Modeling Analysis and Simulation of Wireless and Mobile Systems,2005.

[3] V. Kanodia, A. Sabharwal, and E. Knightly,"MOAR: A multi-channel opportunistic auto-rate media access protocol for ad hoc networks," in Proceedings of IEEE BROADNETS, 2004.

[4] A. Adya, P. Bahl, J. Padhye, A. Wolman, and L. Zhou., "A multi-radio unification protocol for IEEE 802.11 wireless networks", in Proceedings of IEEE BROADNETS, 2004.

[5] A. Nasipuri, J. Zhuang and S.R. Das, "A multichannel CSMA MAC protocol for multihop wireless networks", in Wireless Communications and Networking Conference, 1999
[6] N. Choi, Y. Seok and Y. Choi, "Multi-channel MAC protocol for mobile ad hoc networks", in Vehicular Technology Conference, 2003.

[7] H. Zhai, J. Wang, Y. Fang, D. Wu, "A dualchannel MAC protocol for mobile ad hoc networks", in Global Telecommunications Conference Workshops, 2004.

[8] M. Cao, V. Raghunathan, P.R.Kumar,"Cross-Layer Exploitation of MAC Layer Diversity in Wireless Networks", in Proceedings of ICNP06, The 14th IEEE International Conference on Network Protocols, November 12-15, 2006, Santa Barbara, CA.

[9] R. Knopp and P. A. Humblet, "Information capacity and power control in a single cell multiuser environment", inProceedings of IEEE ICC 1995, Seattle, WA, 1995.

[10] S.L. Wu, C.Y Lin, Y.C. Tseng, J.-P. Sheu, "A New Multi-Channel MAC Protocol with On-Demand Channel Assignment for Multi-Hop Mobile Ad Hoc Networks", in International Symposium on Parallel Architectures, Algorithms and Networks (ISPAN '00), 2000.

[11] J So, Vaidya, "MultiChannel MAC for Ad Hoc Networks: Handling MultiChannel Hidden Terminals Using A Single Transceive", in MobiHoc ,2004

[12] J. Shi, T. Salonidis, Edward W. Knightly, "Medium access control: Starvation mitigation through multi-channel coordination in CSMA multi-hop wireless networks", in MobiHoc,2006.

[13] P. Bhagwat, P. Bhattacharya, A. Krishna and S. K. Tripathi, "Enhancing throughput over wireless LANs using channel state dependent packet scheduling", in Proc. of INFOCOM, 1996.

[14] J. Wang and H. Zhai, "OMAR:Utilizing Multiuser Diversity in Wireless Ad Hoc Networks", IEEE Transactions on mobile computing, 2006.

[15] H. Zhai and Y. Fang, "Physical Carrier Sensing and Spatial Reuse in Multirate and Multihop Wireless Ad Hoc Networks", inProc. of The IEEE International Conference on Computer Communications (INFOCOM'06), Barcelona, Spain, April 23-29, 2006

[16] http://research.microsoft.com/mesh/

[17] http://www.strixsystems.com

[18] http://www.meshdynamics.com/

[19] Feng Chen, "Joint channel and interface assignment in opportunistic MAC", technical report, 2007 